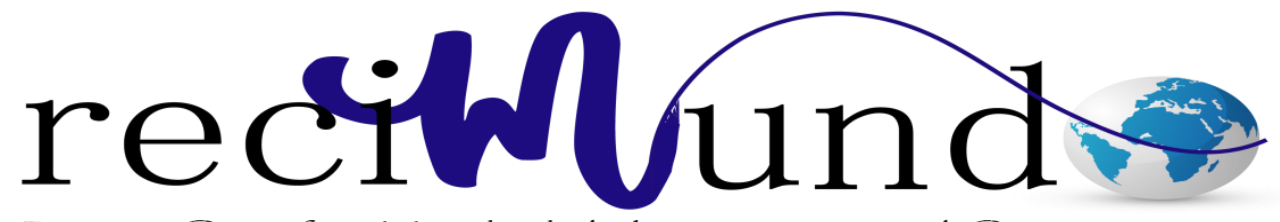

Revista Científica Mundo de la Investigación y el Conocimiento

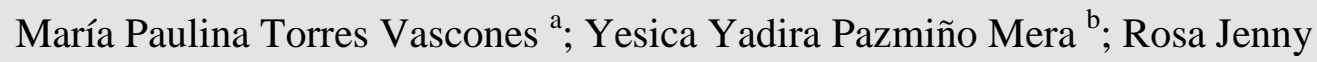 \\ Jumbo Rosillo ${ }^{\text {c }}$ : Miguel Augusto Gonzales Pluas ${ }^{\text {d }}$
}

Riesgos psicosociales y su influencia en niños y adolescentes en conflicto con la ley

Revista Científica Mundo de la Investigación y el Conocimiento. Vol. 2 núm.2, mayo, ISSN: 2588-073X, 2018, pp. 722-741

DOI: 10.26820/recimundo/2.(2).2018.722-741

Editorial Saberes del Conocimiento

Recibido: 05/12/2017 Aceptado: $15 / 02 / 2018$
a. Universidad de Guayaquil; maria.torresv@ug.edu.ec
b. Universidad de Guayaquil; yesica.pazminom@ug.edu.ec
c. Universidad de Guayaquil; rosa.jumbor@ug.edu.ec
d. miguelgonzalespl@hotmail.com 


\section{Riesgos psicosociales y su influencia en niños y adolescentes en conflicto con la}

Vol. 2, núm. 2., (2018)

María Paulina Torres Vascones; Yesica Yadira Pazmiño Mera; Rosa Jenny Jumbo Rosillo;

Miguel Augusto Gonzales Pluas

\section{RESUMEN}

Este proyecto de investigación se basa en estudios realizados en USA. Los centros de detención juvenil, no han resuelto, en su totalidad, los problemas que tienen, diariamente, los oficiales de seguridad y los jóvenes en conflicto con la ley.

El proyecto tratará de crear protocolos adecuados para resolver y aliviar los rasgos psicosociales en oficiales y adolescentes en conflicto con la ley.

Palabras clave: Riesgos psicosociales; psicosociales; violencia laboral. 


\section{Riesgos psicosociales y su influencia en niños y adolescentes en conflicto con la}

ley

Vol. 2, núm. 2., (2018)

María Paulina Torres Vascones; Yesica Yadira Pazmiño Mera; Rosa Jenny Jumbo Rosillo; Miguel Augusto Gonzales Pluas

\section{ABSTRACT}

This research project is based on studies conducted in the USA. The juvenile detention centers have not resolved, in their entirety, the problems that security officers and young people in conflict with the law face on a daily basis.

The project will try to create adequate protocols to solve and alleviate psychosocial traits in officers and adolescents in conflict with the law.

Key words: Psychosocial risks; psychosocial labor violence. 


\section{Riesgos psicosociales y su influencia en niños y adolescentes en conflicto con la

Vol. 2, núm. 2., (2018)

María Paulina Torres Vascones; Yesica Yadira Pazmiño Mera; Rosa Jenny Jumbo Rosillo;

Miguel Augusto Gonzales Pluas

\section{Introducción.}

Este proyecto de investigación se basa en estudios realizados en USA. Los centros de detención juvenil, no han resuelto, en su totalidad, los problemas que tienen, diariamente, los oficiales de seguridad y los jóvenes en conflicto con la ley.

El proyecto tratará de crear protocolos adecuados para resolver y aliviar los rasgos psicosociales en oficiales y adolescentes en conflicto con la ley.

\section{Antecedentes}

El estudio de los aspectos organizacionales y psicosociales y su relación con la salud laboral, no es nuevo, aunque si la importancia y reconocimiento que ha adquirido en los últimos años como consecuencia de los importantes cambios en las organizaciones, y, de los procesos de globalización actual, considerando también la exposición a los riesgos psicosociales, se ha hecho más frecuente e intensa, haciendo conveniente y necesario su identificación, evaluación y control, con el fin de evitar sus riesgos asociados para la salud, y la seguridad en el trabajo.

\section{Importancia y justificación del estudio}

El trabajar en un centro de atención integral con jóvenes y adolescentes es un trabajo fatigante en el turno nocturno, acompañado de un descanso diurno poco reparador, es una de las causas de los trastornos en los trabajadores. Durante el día nuestros parámetros biológicos tienen unas constantes naturales (temperatura, frecuencia cardiaca, consumo de oxígeno, etc.) que son diferentes a las de la noche. 


\section{Riesgos psicosociales y su influencia en niños y adolescentes en conflicto con la ley}

Vol. 2, núm. 2., (2018)

María Paulina Torres Vascones; Yesica Yadira Pazmiño Mera; Rosa Jenny Jumbo Rosillo; Miguel Augusto Gonzales Pluas

Estas extensas jornadas de trabajo y en algunos casos de gran responsabilidad, como es el caso de los oficiales que mantienen la seguridad del centro de detención juvenil, o centro de atención integral, pueden ser factores determinantes en el trabajador al momento de tomar una decisión o de reaccionar ante una emergencia, debido a que puede afectar de la siguiente manera en el trabajador:

Alteraciones del equilibrio biológico, dificultades para mantener atención, trastornos del humor y carácter, trastornos del sueño, trastornos somáticos (cefaleas).

\section{Elementos teóricos que fundamentan la investigación}

La violencia es probablemente un rasgo de las nuevas formas y estilos de vida, predominantemente urbana, anónima, acelerada y competitiva.

La OIT (2003) define la violencia laboral como toda acción, incidente o

Comportamiento que se aparta de lo razonable en la cual una persona es asaltada, amenazada, humillada o lesionada como consecuencia directa de su trabajo.

Este estudio intenta elaborar protocolos y guía para establecer servicios de salud y bienestar mental, así como también, abuso de sustancias, y seguridad laboral, promoviendo seguridad pública, sensibilidad cultural y que sea accesible a adolescentes que requieren de este tipo de servicios.

Muy pocos estudios se han realizado, preocupado, y tratado el problema para mejorar la calidad de vida de los niños, niñas, adolescentes y personal de seguridad, que atienden y 


\section{Riesgos psicosociales y su influencia en niños y adolescentes en conflicto con la

Vol. 2, núm. 2., (2018)

María Paulina Torres Vascones; Yesica Yadira Pazmiño Mera; Rosa Jenny Jumbo Rosillo;

Miguel Augusto Gonzales Pluas

supervisan por el bienestar de los jóvenes residentes y que están en conflicto con la ley. En un centro de atención integral. El centro estará administrado bajo un sistema estructurado en educación, psicoterapia, abuso y dependencia de substancias, y conductas disruptivas.

Como se puede visualizar, es un programa ambicioso y que en USA ya están funcionando y cada vez van revisando sus objetivos para ver si se han cumplido; no obstante falta mucho por hacer, el abuso de drogas y alcohol persiste y la violencia está presente en escuelas, colegios y hogares.

Es importante resaltar el trabajo que realizan los oficiales de seguridad para lograr cumplir con los objetivos planteados. Ellos son los que tienen que resolver y controlar los problemas de comportamiento y violencia que presentan estos jóvenes con desórdenes psicológicos. Así mismo, los oficiales de seguridad son quienes tienen que liderar para que los residentes experimenten un sistema estructurado, donde existe horarios de dormir, despertar, desayunar, ir a clases, almorzar, realizar actividades físicas, y recreativas.

Tienen que lidiar a diario con grupos de 40 O más adolescentes, con una variedad de trastornos violentos y conductas disruptivas. Los oficiales, por estas razones, sufren mucho de estrés laboral, dolencias y dolores musculares, Uso y abuso de alcohol, violencia doméstica.

Como consecuencia de todo ello, la segunda encuesta europea de calidad de vida indica que casi la mitad de los ciudadanos con trabajo remunerado en los 27 países de la UE reconocen que varias veces al mes están demasiado cansados para hacer las tareas del hogar, y el $22 \%$ afirman estarlo varias veces a la semana. 


\section{Riesgos psicosociales y su influencia en niños y adolescentes en conflicto con la ley}

Vol. 2, núm. 2., (2018)

María Paulina Torres Vascones; Yesica Yadira Pazmiño Mera; Rosa Jenny Jumbo Rosillo; Miguel Augusto Gonzales Pluas

El $11 \%$ refiere que tiene dificultades varias veces por semana para llevar a cabo las responsabilidades familiares por el tiempo que pasan en el trabajo.

http://www.eurofound.europa.eu/pubdocs/2009/02/ en/2/EF0902EN.pdf

Un entorno de trabajo saludable y seguro es la mejor garantía del rendimiento laboral, de la salud de sus empleados y de la motivación e implicación organizacional. La pérdida de calidad del trabajo entraña costes a veces difíciles de observar a corto plazo, pero siempre presentes a medio y largo plazo.

Es un trabajo estresante para el personal que labora y tiene contacto directo con los jóvenes detenidos. Por esta razón, se sugieren los siguientes objetivos para prevenir el agotamiento del personal en contacto directo con los jóvenes en conflicto.

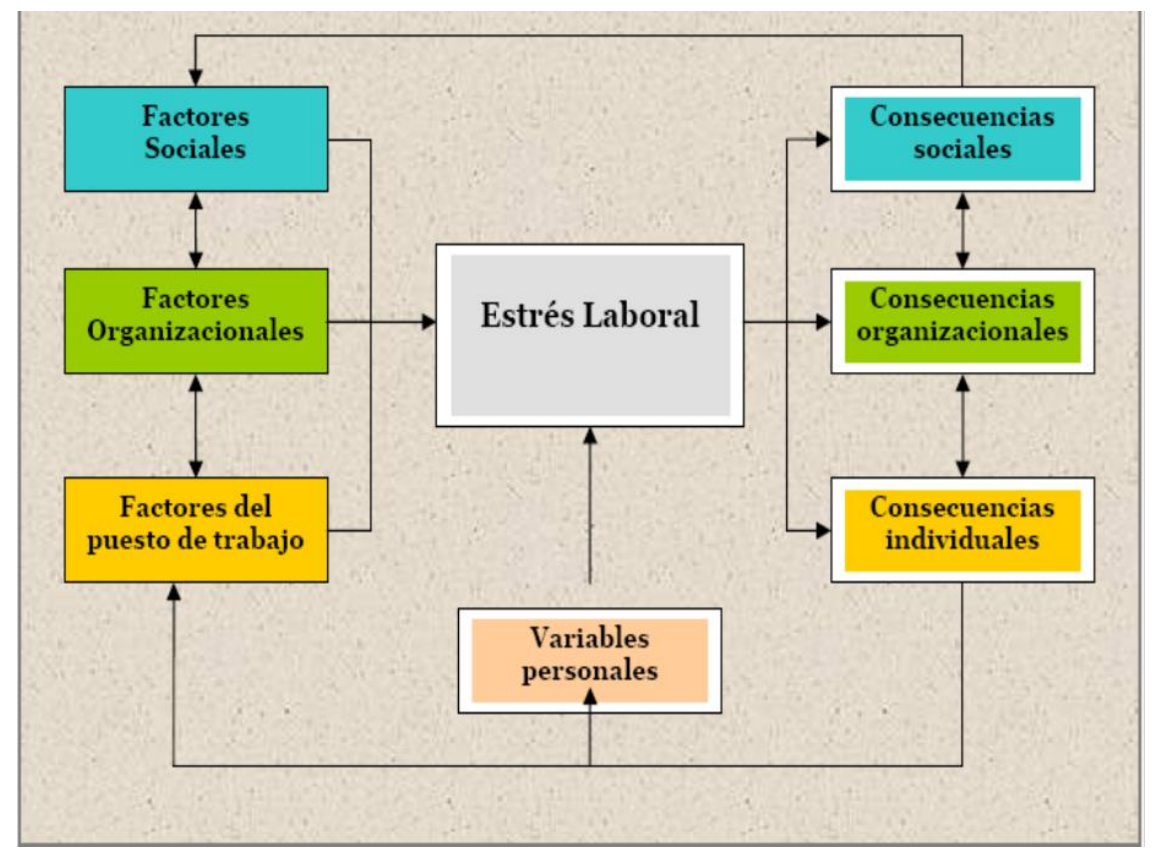




\section{Riesgos psicosociales y su influencia en niños y adolescentes en conflicto con la

Vol. 2, núm. 2., (2018)

María Paulina Torres Vascones; Yesica Yadira Pazmiño Mera; Rosa Jenny Jumbo Rosillo;

Miguel Augusto Gonzales Pluas

\section{Generalidades}

Acoso laboral

El mobbing o acoso laboral es considerado actualmente uno de los riesgos laborales más importantes en la vida laboral.

Los efectos de los atentados a la propia dignidad e intimidad del trabajador no tienen ni la misma forma, ni la misma naturaleza, ni las mismas consecuencias, que puede tener un atraco o asalto o la violencia física.

\section{Acoso sexual}

El acoso sexual es planteado también como una de las formas de la violencia laboral, y más frecuentemente como una modalidad del acoso laboral, sin embargo, el acoso sexual tiene igualmente un contexto propio, unas formas específicas, y unas consecuencias especiales, que hace que no se identifique con la violencia en el trabajo o el acoso laboral.

\section{Inseguridad contractual}

La inseguridad laboral podría definirse como una preocupación general acerca de la existencia del trabajo en el futuro, y también como una amenaza percibida de características de trabajo diferentes, tales como la posición dentro de una organización o las oportunidades de carrera. 


\section{Riesgos psicosociales y su influencia en niños y adolescentes en conflicto con la ley}

Vol. 2, núm. 2., (2018)

María Paulina Torres Vascones; Yesica Yadira Pazmiño Mera; Rosa Jenny Jumbo Rosillo; Miguel Augusto Gonzales Pluas

\section{El Burn Out o Desgaste Profesional}

Consiste en el resultado de un proceso de estrés crónico laboral y organizacional que termina en un estado de agotamiento emocional y de fatiga desmotivarte para las tareas laborales.

- Salud: No solamente significa la ausencia de enfermedades, accidentes o discapacidades, sino un estado óptimo de bienestar físico, psíquico y social.

- Peligro: es cualquier cosa que puede causar daño.

- Riesgo: La probabilidad de que alguien sea lesionado por el peligro.

La adaptación es extremadamente importante para el futuro laboral de los oficiales de estos centros; es la capacidad de dos realidades para complementarse entre sí. Ejemplo, el oficial y su entorno laboral.

Estrés: Es una desadaptación del ser humano frente a su ambiente, puede durar poco o varios días. Cada oficial reacciona diferente frente al estrés, aún cuando las causas sean las mismas; todos los seres humanos padecen de estrés en algún momento de sus vidas.

\section{Mobbing (Acoso profesional)}

En el Congreso sobre Higiene y Salud en el Trabajo del año de 1990, el psicólogo sueco Heinz Leymann, definió por primera vez el término mobbing como "la situación en que una persona ejerce una violencia psicológica extrema de forma sistemática, recurrente y durante un tiempo prolongado sobre otra persona o personas en el lugar de trabajo, con la finalidad de 


\section{Riesgos psicosociales y su influencia en niños y adolescentes en conflicto con la

Vol. 2, núm. 2., (2018)

María Paulina Torres Vascones; Yesica Yadira Pazmiño Mera; Rosa Jenny Jumbo Rosillo;

Miguel Augusto Gonzales Pluas

destruir las redes de comunicación de la víctima o víctimas, destruir su reputación, perturbar el ejercicio de sus labores y lograr que esa persona acabe abandonando el lugar de trabajo".

Indudablemente que el concepto de Leymann, y, en sus posteriores estudios se refleja una extrema violencia laboral, donde vamos a destacar no solo la posible agresión física, que es más visible, sino, la agresión verbal Y mental que tiene negativas repercusiones en la conducta de los agredidos.

En USA, se realizó un estudio, donde se identificó que si existía acoso sexual en los centros de detención juvenil; el estado actuó inmediatamente, y las sanciones fueron radicales.

Causas del estrés:

Causas Externas:

Psicosociales: conflictos, apuros económicos, pérdida o cambio de trabajo, pérdidas personales, presión de tiempo, presión de trabajo, jubilación, desengaños, clima, ruido.

\section{Psicofisiologìa del estrés}

Fase de alarma: El cuerpo se prepara para combatir la amenaza y Aumenta significativamente el consumo de energía.

Fase de acción: El cuerpo ejecuta las acciones necesarias para eliminar la amenaza o peligro.

Fase de relajamiento: El cuerpo retorna a su equilibrio (físico - mental). 


\section{Riesgos psicosociales y su influencia en niños y adolescentes en conflicto con la ley}

Vol. 2, núm. 2., (2018)

María Paulina Torres Vascones; Yesica Yadira Pazmiño Mera; Rosa Jenny Jumbo Rosillo; Miguel Augusto Gonzales Pluas

Bajan los niveles de energía. Siente alivio físico.

\section{Metodología.}

El MBI (Maslach de 1986)

- $\quad$ Es el instrumento más utilizado para levantar información del BURNOUT.

- Este cuestionario tiene una alta consistencia interna y una fiabilidad cercana al $90 \%$.

- Está constituido por 22 ítems en forma de afirmaciones, sobre los sentimientos y actitudes del profesional en su trabajo y hacia los pacientes; su función es medir el desgaste profesional.

Què mide?

- Cansancio emocional;

- Describen sentimientos de estar abrumado y agotado Emocionalmente por el trabajo. 1-2-3-6-8-13-14-16-20;

- Despersonalización;

- Describen una respuesta impersonal y falta de sentimientos hacia los sujetos objeto de atención. 5-10-11-15-22;

- $\quad$ Realización personal; 


\section{Riesgos psicosociales y su influencia en niños y adolescentes en conflicto con la

Vol. 2, núm. 2., (2018)

María Paulina Torres Vascones; Yesica Yadira Pazmiño Mera; Rosa Jenny Jumbo Rosillo;

Miguel Augusto Gonzales Pluas

Describen sentimientos de competencia y realización exitosa en el trabajo hacia los demás. $4-7-9-1$.

\section{Resultados}

Respuesta anormal ante el estrés

También conocido como Síndrome de adaptación general. Se puede apreciar la presencia de signos y síntomas concurrentes de tipo físico, mental y de comportamiento. El personal de los centros de detención juvenil suelen llevar sus problemas a sus hogares; existe la posibilidad de reaccionar violentamente ante cualquier situación con sus familiares; el abuso del alcohol, también es común.

\section{Fase de resistencia:}

Adaptación: el cuerpo trata de adaptarse y reparar los daños; existe tensión y dolor muscular (cuello, hombros, espalda); Se experimenta fatiga frecuente; molestias en el pecho. Puede experimentar desórdenes digestivos, insomnio y pesadillas.

Hay tendencia a la irritabilidad, miedos, fobias, ansias por comer algo; falta de concentración.

\section{Fase de agotamiento:}

Se rompe el equilibrio y se produce alteraciones en uno o más sistemas u órganos.

El organismo tiende a colapsar y ceder ante la enfermedad, se ha notado que el personal de seguridad en los centros puede experimentar hipertensión arterial, asma, migraña. 


\section{Riesgos psicosociales y su influencia en niños y adolescentes en conflicto con la ley}

Vol. 2, núm. 2., (2018)

María Paulina Torres Vascones; Yesica Yadira Pazmiño Mera; Rosa Jenny Jumbo Rosillo; Miguel Augusto Gonzales Pluas

\section{Consecuencias del estrés crónico:}

Gastritis, frustración, insomnio, colitis nerviosa, migraña, crisis de pánico, alteraciones sexuales, problemas laborales, adicciones, conductas antisociales, psicosis, sarpullidos, arritmias cardíacas, mareos.

Por todo lo expuesto anteriormente, los centros de detención juvenil en USA, han puesto en marcha diferentes mecanismo para aliviar, de alguna manera, el agotamiento laboral y turnicidad de los oficiales, ya que los centros de detención juvenil funcionan 24 horas al día 365 días del año, en otras palabra, no existen feriados, los jóvenes en detención no pueden quedarse sin supervisión en ningún momento. Los oficiales, definitivamente, tienen que trabajar todo el tiempo, en diferentes turnos y horarios.

\section{Riesgos turnos de trabajo:}

Alteraciones del equilibrio biológico, dificultades para mantener atención, trastornos del humor y carácter, trastornos del sueño, trastornos somáticos.

1. Alteraciones de la vida social.- El trabajo a turnos, especialmente el de la noche y el de la tarde, dificulta las relaciones sociales debido a la falta de coincidencia con los demás;

2. Incidencia en la actividad profesional, La baja actividad del organismo durante la noche, puede provocar que se den una serie de percusiones negativas sobre la ejecución del trabajo (actuar con rapidez, percibir correctamente la información);

3. Problemas y enfermedades cardiovasculares; 


\section{Riesgos psicosociales y su influencia en niños y adolescentes en conflicto con la}

Vol. 2, núm. 2., (2018)

María Paulina Torres Vascones; Yesica Yadira Pazmiño Mera; Rosa Jenny Jumbo Rosillo;

Miguel Augusto Gonzales Pluas

4. Depresión, ansiedad y otros trastornos de salud mental;

5. Dolor de espalda y otros trastornos músculo esquelético;

6. Trastornos respiratorios y gastrointestinales;

7. Cambio de Conductas sociales (cigarrillo, drogas, sedentarismo, falta de participación social);

8. Absentismo laboral;

Los factores psicosociales cuando son factores con probabilidad de afectar negativamente a la salud y el bienestar del trabajador son factores de riesgo (Benavides et al. 2002), es decir, cuando actúan como factores desencadenantes de tensión y de estrés laboral (Peiró,1993);

Los accidentes y errores son más frecuentes en trabajadores con problemas de auto adaptación.

\begin{tabular}{|c|c|}
\hline \multicolumn{2}{|c|}{ Consecnencias $y$ efectos de los riesgos psicosociales } \\
\hline \multicolumn{2}{|c|}{ 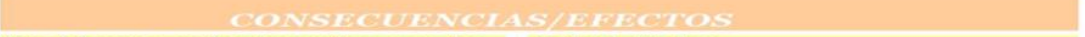 } \\
\hline $\begin{array}{l}\text { Problemas relacionados con la } \\
\text { salud }\end{array}$ & $\begin{array}{l}\text { Salud Física } \\
\text { Salud Mental } \\
\text { Consumo de sustancias } \\
\text { Trastornos psicosomáticos }\end{array}$ \\
\hline Actitudes ante la empresa & $\begin{array}{l}\text { Satisfacción laboral } \\
\text { Implicación laboral } \\
\text { Conductas contraproducentes }\end{array}$ \\
\hline Tiempos de trabajo & $\begin{array}{l}\text { Rotación de personal } \\
\text { Presentismo } \\
\text { Bajas laborales } \\
\text { Duración de las haias }\end{array}$ \\
\hline Costes económicos & $\begin{array}{l}\text { Accidentes de trabajo } \\
\text { Perdida de materiales } \\
\text { Rendimiento } \\
\text { Productividad }\end{array}$ \\
\hline
\end{tabular}




\section{Riesgos psicosociales y su influencia en niños y adolescentes en conflicto con la ley}

Vol. 2, núm. 2., (2018)

María Paulina Torres Vascones; Yesica Yadira Pazmiño Mera; Rosa Jenny Jumbo Rosillo; Miguel Augusto Gonzales Pluas

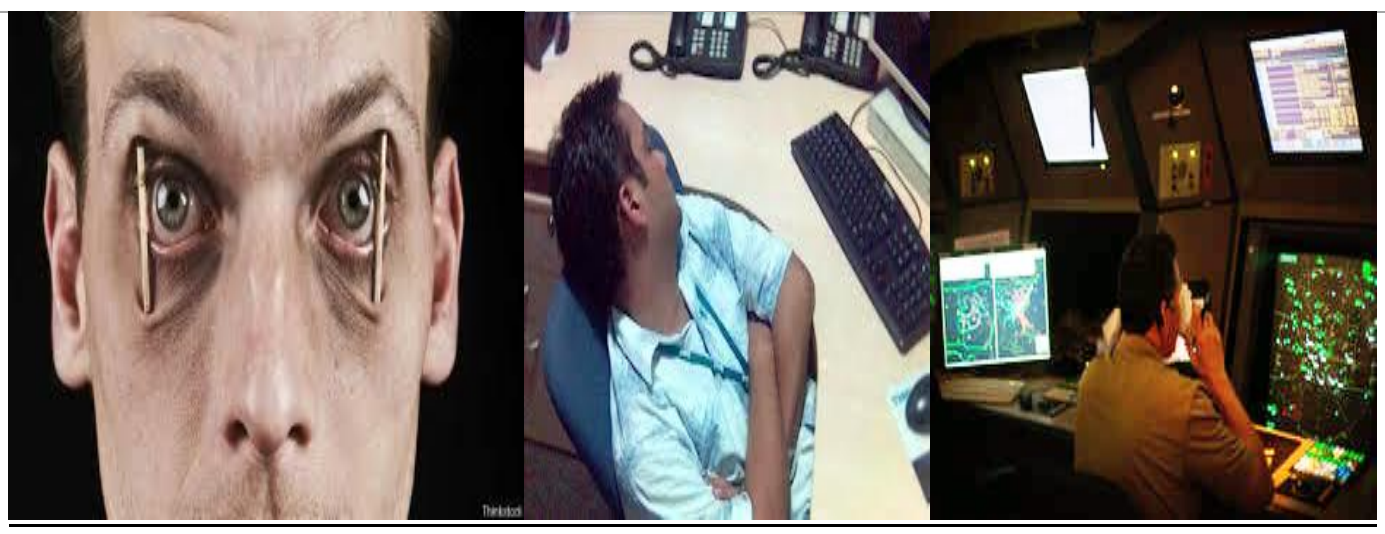

\section{Conclusiones}

El Director del centro debe participar, activamente, con la policía nacional, escuelas y colegios para prevenir la participación pandillera dentro y fuera de las instituciones de educación.

Debe estar familiarizado con las diferentes pandillas existentes en las calles de cualquier ciudad y país. Existen varias formas para determinar presencia de actividad pandillera.

Los oficiales deben mantenerse alerta, ya que los jóvenes, miembros de una pandilla, tienden a ser agresivos, violentos y sobre todo, tratan de intimidar a otros residentes del centro, y, por supuesto, a oficiales, especialmente, cuando no son experimentados o son oficiales nuevos en el oficio. Por esta razón, es que en USA, los oficiales, tienen que asistir a la Academia de Policía, (4 semanas) para recibir clases y entrenamiento de cómo actuar bajos estas presiones conductuales y laborales.

No hay que olvidar que estos jóvenes pueden ser bastante difíciles ya que no tienen estructurada su vida, y a veces carecen de amor y empatía desde sus hogares, o sencillamente, no tienen hogar 


\section{Riesgos psicosociales y su influencia en niños y adolescentes en conflicto con la

Vol. 2, núm. 2., (2018)

María Paulina Torres Vascones; Yesica Yadira Pazmiño Mera; Rosa Jenny Jumbo Rosillo;

Miguel Augusto Gonzales Pluas

más que el de la pandilla. Los siguientes puntos son cruciales para que los oficiales de seguridad y empleados del centro tengan en cuenta:

1. Grafiti: es uno de los métodos más comunes para determinar la presencia de pandillas. Esto incluye el uso de símbolos como la corona de tres puntas, la estrella de cinco puntas, etc. Así como también, el uso de escritos crípticos; usualmente, un alfabeto modificado. A menudo se pueden apreciar estos signos en trabajos de arte hecho por los residentes.

2. Apariencia personal: Puede proveer al personal de seguridad y empleados ciertas pistas acerca de una posible actividad pandillera. Por ejemplo, los residentes pueda que tengan un corte de cabello que son únicos o comunes con afiliaciones pandilleras, o pueda que quieran usar su ropa de una manera exclusiva, como son los pantalones envueltos hacia arriba de una sola pierna; uso de bandanas, y colores exclusivos de ciertas pandillas.

3. Comunicación críptica: es otro método de comunicación para determinar actividad pandillera, los miembros de estas pandillas usan slang terms; uso de signos con las manos ( flashing), incluyendo el uso para irrespetar rivales pandilleros y crear una posible pelea. Por ejemplo, el uso de la letra c cruzada es un irrespeto hacia los crips. 


\section{Riesgos psicosociales y su influencia en niños y adolescentes en conflicto con la ley}

Vol. 2, núm. 2., (2018)

María Paulina Torres Vascones; Yesica Yadira Pazmiño Mera; Rosa Jenny Jumbo Rosillo; Miguel Augusto Gonzales Pluas
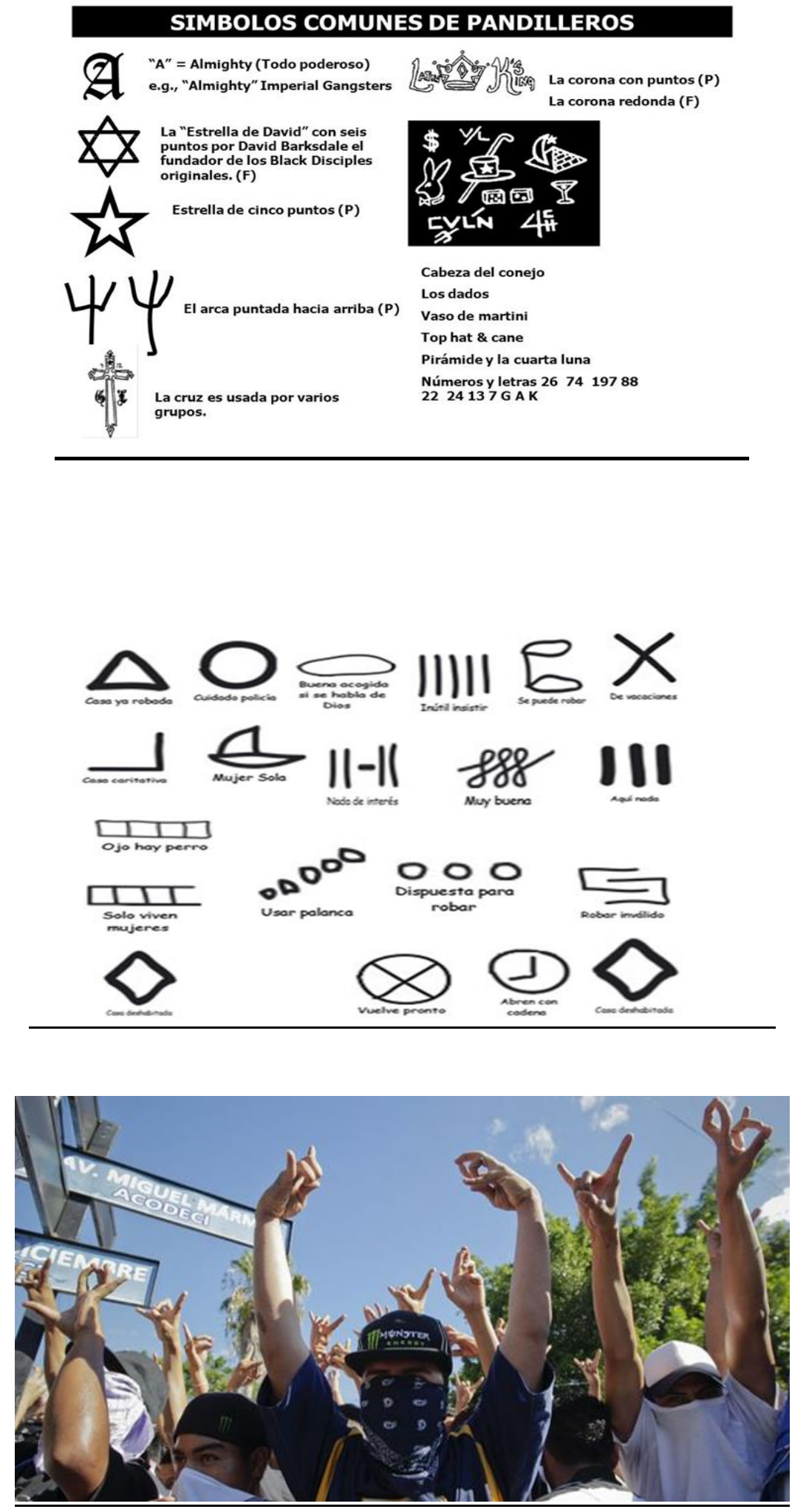
Riesgos psicosociales y su influencia en niños y adolescentes en conflicto con la ley

Vol. 2, núm. 2., (2018)

María Paulina Torres Vascones; Yesica Yadira Pazmiño Mera; Rosa Jenny Jumbo Rosillo;

Miguel Augusto Gonzales Pluas
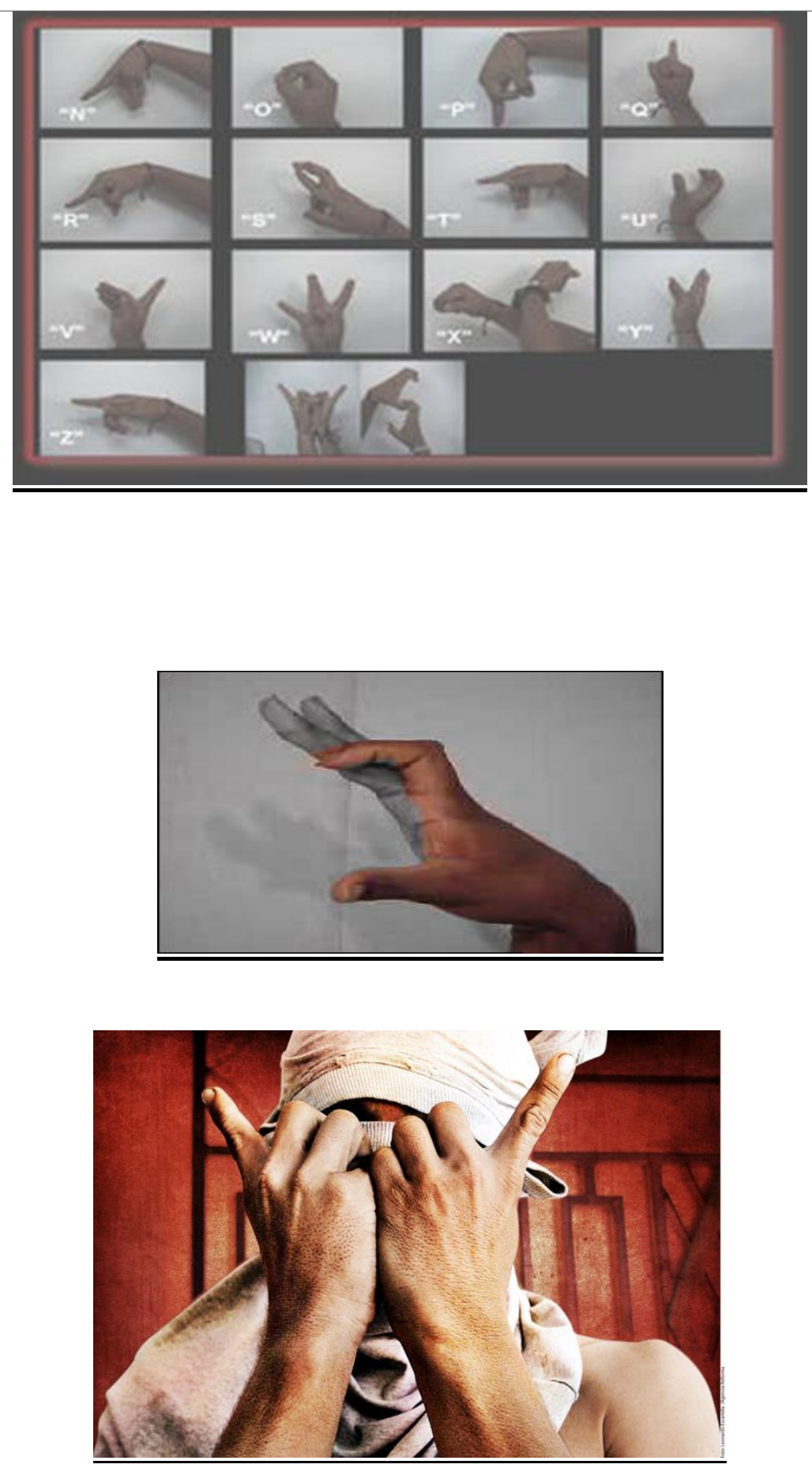


\section{Riesgos psicosociales y su influencia en niños y adolescentes en conflicto con la ley}

Vol. 2, núm. 2., (2018)

María Paulina Torres Vascones; Yesica Yadira Pazmiño Mera; Rosa Jenny Jumbo Rosillo; Miguel Augusto Gonzales Pluas

\section{Protección y acción responsable (PAR)}

Todos los supervisores de disciplina del centro son requeridos conocer y usar PAR cuando se amerite el caso. PAR es utilizado como método de intervención para:

1. Mantener seguridad y un ambiente seguro para los residentes, personal administrativo, médico, psicólogos, enfermeras y mantenimiento.

2. Cuidado efectivo de seguridad.

3. Brinda seguridad al público.

4. Mantiene disciplina.

Para lograr el uso adecuado de seguridad mediante el uso de PAR, los oficiales del centro tienen que tomar clases y aprobar el examen para obtener su respectiva certificación. Esta certificación debe ser renovada cada año para prevenir el uso indebido con los jóvenes de estos centros.

Como referencia, se puede decir que en USA, los aspirantes a oficiales de los centros de detención, deben obtener la certificación de cada estado, mediante un curso intensivo de 3 a 4 semanas donde estudian lo que deben hacer en cada paso durante una emergencia. No hay que olvidar que los adolescentes y niños son frágiles y pueden sufrir daños físicos.

\section{Bibliografía.}

Caballero J., 2005, “Acoso psicológico en el trabajo: mobbing”, Revista Paceña de medicina familiar. 


\section{Riesgos psicosociales y su influencia en niños y adolescentes en conflicto con la}

Vol. 2, núm. 2., (2018)

María Paulina Torres Vascones; Yesica Yadira Pazmiño Mera; Rosa Jenny Jumbo Rosillo;

Miguel Augusto Gonzales Pluas

Centro nacional de condiciones de trabajo, 2006 "Acoso psicológico en el trabajo: mobbing", España.

León M., Muñiz I., Martín L.2012 "El acoso psicológico en el trabajo o mobbing: patología emergente", Valladolid-España,

Moreno B., Rodríguez A., Morante M., 2008, Garro "Evaluación del acoso psicológico en el trabajo: desarrollo y estudio exploratorio de una escala de medida", Madrid-España. 